\title{
Observed characteristics of tropical cyclone vertical wind profiles
}

\author{
Ian M. Giammanco*1, John L. Schroeder ${ }^{1}$ and Mark D. Powell ${ }^{2}$ \\ ${ }^{1}$ Wind Science and Engineering Research Center, Texas Tech University, Lubbock, Texas USA \\ ${ }^{2}$ NOAA/AOML Hurricane Research Division, Miami, Florida, USA \\ (Received March 2, 2011, Revised June 15, 2011, Accepted July 7, 2011)
}

\begin{abstract}
Over the last decade substantial improvements have been made in our ability to observe the tropical cyclone boundary layer. Low-level wind speed maxima have been frequently observed in Global Positioning System dropwindsonde (GPS sonde) profiles. Data from GPS sondes and coastal Doppler radars were employed to evaluate the characteristics of tropical cyclone vertical wind profiles in open ocean conditions and at landfall. Changes to the mean vertical wind profile were observed azimuthally and with decreasing radial distance toward the cyclone center. Wind profiles within the hurricane boundary layer exhibited a logarithmic increase with height up to the depth of the wind maximum.
\end{abstract}

Keywords: tropical cyclones; GPS dropwindsonde; radar; wind; profiles; low-level jets; velocity azimuth display.

\section{Introduction}

Tropical cyclones are one of nature's most violent events and have the capacity to inflict significant damage over a large swath of coastline. Property loses due to landfalling hurricanes have increased dramatically (Pielke et al. 2008). As coastal populations continue to grow, the need to understand the variability in the wind field of a landfalling hurricane both horizontally and vertically has become apparent. As construction of large high-rise structures continues along hurricane-prone coastlines, the understanding of the characteristics of the vertical wind profile is sorely needed. Additionally, validation is needed with regards to the use of logarithmic and power law relationships to represent the profile within structural design standards. Understanding the structure and stormrelative location of low-level wind maxima may also provide information on expected surface gust factors. Wind maxima aloft may represent a source of momentum available for transport to the surface.

The implementation of the Global Positioning System dropwindsonde (GPS sonde) has provided a wealth of high resolution wind profiles within tropical cyclones (Hock and Franklin 1999). Observations obtained from GPS sondes were employed to characterize the vertical wind profile in open ocean conditions. Unfortunately a significant gap remains in historical observations, with only minimal information available from hurricanes at landfall. The coastal network of Weather

\footnotetext{
* Corresponding author, E-mail: ian.giammanco@ttu.edu
} 
Surveillance Radars (WSR-88D) operated by the National Weather Service (NWS) provided a means to fill the data void at landfall (Klazura and Imy 1993).

GPS sonde observations have revealed the presence of low-level wind speed maxima at heights well below $1 \mathrm{~km}$ above ground level, near the top of the hurricane boundary layer (HBL). GPS sonde composite wind profiles contained a jet-like structure with an increase in wind speed to a maximum near $500 \mathrm{~m}$, followed by a gradual decrease (Franklin et al. 2003, Powell et al. 2003). Powell et al. (2003) found a more pronounced jet-like profile. It was also found that the wind profile within a specified surface layer was logarithmic. Vickery et al. (2009) provided evidence, using a similar dataset, that the profile remained logarithmic up to the height of the wind speed maximum. Studies by Kepert (2006 a,b) and Schwendike and Kepert (2008) found that the vertical wind profile is subject to storm by storm variability associated with dynamic processes of hurricanes. Individual GPS sonde wind profiles often exhibit multiple local wind speed maxima and minima owing to the high resolution measurement characteristics of the instrument.

Vertical wind profile observations of the HBL from the coastal region during hurricane landfalls are scarce relative to those found in open ocean conditions due to restrictions on reconnaissance aircraft. The coastal transition of the wind field as the flow moves from deep water to shallow water to land surface, complicates the vertical structure of the HBL. Internal boundary layer (IBL) processes influence the shape of the vertical profile in this region. Blackwell (2000) and Knupp et al. (2006) have shown some observational evidence of jet-like wind maxima at altitudes near $500 \mathrm{~m}$ in two weak landfalling tropical cyclones. A preference for the off-shore flow regime was noted.

Validation of assumed logarithmic and power law profiles at landfall has been quite limited. Amano et al. (1999) provided evidence to suggest that power law wind profiles compared well to acoustic profiler observations from several landfalling typhoons. However, these profiles were limited to a relatively shallow vertical extent and did not provide any evidence of low-level wind maxima.

The present study attempts to examine the characteristics of observed low-level wind maxima and their accompanying vertical profiles. A large number of GPS sonde profiles $(>1000)$ were used to assess conditions over the open ocean. A modified velocity azimuth display (VAD) technique was applied to WSR-88D Doppler velocity data to generate over 300 vertical profiles from various landfalling hurricanes over land and near the coast. Profiles were also examined in a storm-relative framework in order to understand radial and azimuthal variability.

\section{GPS sonde data and velocity azimuth display technique}

\subsection{GPS sonde}

The GPS sonde was first deployed into tropical cyclones in 1997 (Hock and Franklin 1999). The instrument package is launched from reconnaissance aircraft at altitudes of $1.5-3 \mathrm{~km}$ and falls at typical speeds between $10-15 \mathrm{~ms}^{-1}$. Measurements of the standard kinematic and thermodynamic variables are made at 0.5 -second intervals. The sampling rate and fall speed yields a vertical resolution of approximately $2 \mathrm{~m}$. An example of a GPS sonde wind profile is shown in Fig. 1. The three-dimensional wind vector is derived from GPS tracking of the instrument package as it descends. Additional information on the wind vector calculation can be found in Hock and Franklin (1999). The typical horizontal wind measurement error was found to be $0.5-2 \mathrm{~ms}^{-1}$ with 

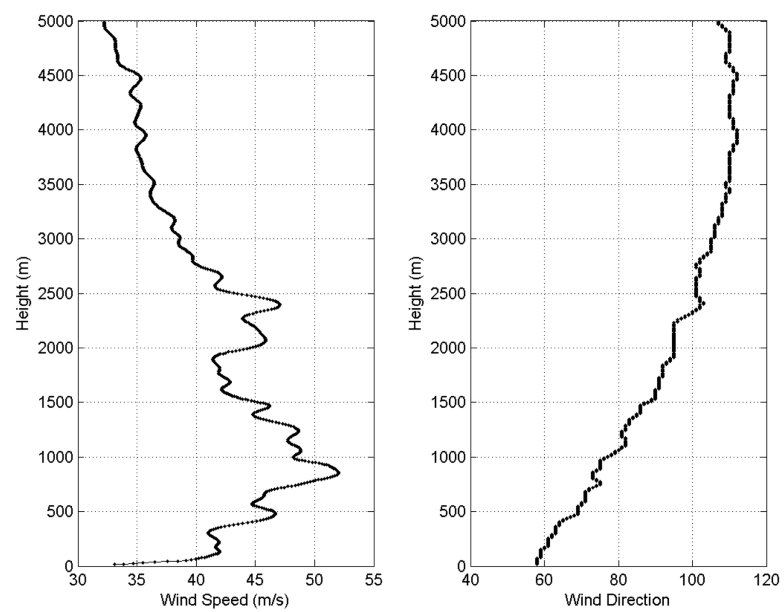

Fig. 1 GPS dropwindsonde profile from Hurricane Rita at 1909 UTC 22 September 2005

an error of nearly $4 \mathrm{~ms}^{-1}$ associated with the vertical wind component (Hock and Franklin 1999). A 5-second low-pass filter is applied to the profile in order to remove under-sampled scales of motion as well as radio frequency noise (Hock and Franklin 1999). The filter results in a vertical filter depth of approximately $50 \mathrm{~m}$. Extreme turbulence and intense precipitation can also contribute to the degradation of the telemetry transmitted by the instrument.

\subsubsection{Data quality control}

The post-processing of GPS sonde data is conducted by the Hurricane Research Division (HRD) of the National Oceanographic and Atmospheric Administration's (NOAA) Atlantic Oceanographic and Meteorological Laboratory (AOML). Sondes which did not transmit data below a given height or exhibit substantial data gaps are often excluded. Wind data quality flags are subjectively assigned to each profile during the review process. Sondes which exceed three flags are removed and no longer considered for post-processing. Between 1997 and 2005, 6148 sondes were released and only 2343 were post-processed by HRD (Powell 2007). For each post-processed GPS sonde a storm track file is assigned. A cubic spline is fit to the observed storm position estimates according to the method described by Willoughby and Chelmow (1982). The high resolution track is used to compute storm-relative quantities such as: azimuth, radius, and radial and tangential wind components. Additional quality control measures were taken for the current study as profiles were restricted to within 2-200 $\mathrm{km}$ of the storm center in order to limit the influence of GPS sondes launched during synoptic surveillance missions and those released very near the cyclone center. GPS sondes which failed to transmit data below $200 \mathrm{~m}$ were also excluded from the analysis dataset. The additional quality control measures resulted in 1080 GPS sondes used for the current study.

\subsection{VAD wind profiles}

Since the deployment of the WSR-88D, there have been numerous tropical cyclone landfalls along the United States coastline. Landfall events in which the cyclone center passed within $150 \mathrm{~km}$ 


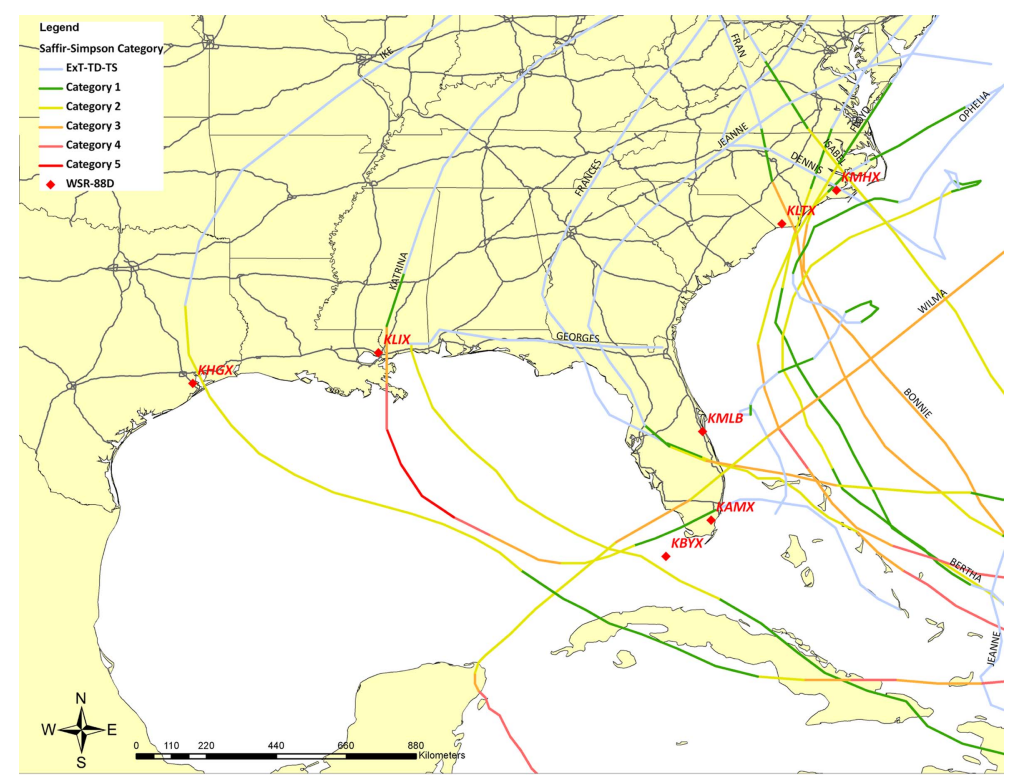

Fig. 2 Best-fit track for all storms and WSR-88D radars used in the VAD analysis

of a WSR-88D site along the Atlantic and Gulf coasts were identified and historical radar data acquired. Radar sites were limited to those within $15 \mathrm{~km}$ of the immediate coastline, a coastal bay, or tidal lake in an effort to obtain data near the shoreline and mitigate the influence of inland decay processes. Fifteen events were selected from 1996-2008. Storms included were of strong tropical storm strength or greater. Fig. 2 provides the best-fit track for each storm included in the dataset as well as the locations of the selected WSR-88D radars. Table 1 lists the landfall events, radar sites, Saffir-Simpson category at the time of analysis, number of profiles used in the present study, general terrain characteristics surrounding each radar site, and the distance of the radar to the closest tidal body of water. The time window of acquired data was subjectively determined and typically began at the onset of outer rainband features and continued through the storm's closest approach to the radar site as identified using the Plan Position Indicator (PPI) reflectivity display. Individual volumes of data were obtained at approximately 15 minute intervals within the domain.

\subsubsection{Modified VAD technique}

The velocity azimuth display technique described by Browning and Wexler (1968) was modified to produce wind profiles of the lowest $1500 \mathrm{~m}$. The method assumes that Doppler velocity measurements vary as a sinusoid with radar azimuth. A Fourier series least-squares fit is applied to the velocity data as a function of azimuth. The corresponding Fourier coefficients $a_{1}$ and $b_{1}$ are used to determine wind speed and direction by

$$
W S=-\frac{\left(a_{1}^{2}+b_{1}^{2}\right)^{\frac{1}{2}}}{\cos \alpha}
$$




$$
\begin{aligned}
& W d=\frac{\pi}{2}-\tan ^{-1} \frac{a_{1}}{b_{1}} \text { for } b<0 \\
& W d=\frac{3 \pi}{2}-\tan ^{-1} \frac{a_{1}}{b_{1}} \text { for } b>0
\end{aligned}
$$

Table 1. List of landfall events, radar sites, total number of profiles generated for each event included in the VAD analysis, and general terrain characteristics within the VAD domain. It is noted that within each VAD domain there are likely transitional flow regimes as several different exposures are typically

\begin{tabular}{|c|c|c|c|c|c|}
\hline Storm & $\begin{array}{l}\text { Radar } \\
\text { site }\end{array}$ & $\begin{array}{l}\text { Saffir- } \\
\text { simpson }\end{array}$ & Profiles & Terrain exposure (N-E-S-W) & $\begin{array}{c}\text { Distance -direction to } \\
\text { immediate shoreline or } \\
\text { tidal lake }\end{array}$ \\
\hline $\begin{array}{l}\text { Hurricane Bertha } \\
\text { (1996) }\end{array}$ & KLTX & 2 & 28 & $\begin{array}{l}\text { Forested-Forested-Sububan- } \\
\text { Forested }\end{array}$ & $11 \mathrm{~km}-\mathrm{SE}$ \\
\hline $\begin{array}{l}\text { Hurricane Fran } \\
\text { (1996) }\end{array}$ & KLTX & 3 & 20 & $\begin{array}{l}\text { Forested-Forested-Subur- } \\
\text { ban-Forested }\end{array}$ & $11 \mathrm{~km}-\mathrm{SE}$ \\
\hline $\begin{array}{l}\text { Hurricane Bonnie } \\
\text { (1998) }\end{array}$ & KLTX & 3 & 2 & $\begin{array}{l}\text { Forested-Forested-Subur- } \\
\text { ban-Forested }\end{array}$ & $11 \mathrm{~km}-\mathrm{SE}$ \\
\hline $\begin{array}{l}\text { Hurricane Georges } \\
\text { (1998) }\end{array}$ & KBYX & 2 & 43 & $\begin{array}{l}\text { Marine-Marine-Marine- } \\
\text { Marine }\end{array}$ & $\begin{array}{l}\text { VAD domain extends } \\
\text { over water }\end{array}$ \\
\hline $\begin{array}{l}\text { Tropical Storm } \\
\text { Dennis (1999) }\end{array}$ & KMHX & TS & 16 & $\begin{array}{l}\text { Suburban-Suburban-For- } \\
\text { ested-Suburban }\end{array}$ & $\begin{array}{l}6 \mathrm{~km} \text { (Bay) - S } \\
10 \mathrm{~km}-\mathrm{S}\end{array}$ \\
\hline $\begin{array}{l}\text { Hurricane Floyd } \\
\text { (1999) }\end{array}$ & KLTX & 2 & 26 & $\begin{array}{l}\text { Forested-Forested-Subur- } \\
\text { ban-Forested }\end{array}$ & $11 \mathrm{~km}-\mathrm{SE}$ \\
\hline $\begin{array}{l}\text { Hurricane Isabel } \\
\qquad(2003)\end{array}$ & KMHX & 2 & 29 & $\begin{array}{l}\text { Suburban-Suburban-For- } \\
\text { ested-Suburban }\end{array}$ & $11 \mathrm{~km}-\mathrm{SE}$ \\
\hline $\begin{array}{l}\text { Hurricane Frances } \\
\qquad(2004)\end{array}$ & KMLB & 2 & 34 & $\begin{array}{l}\text { Suburban- Suburban } \\
\text { /Marine-Suburban-Airport } \\
\text { /Suburban }\end{array}$ & $\begin{array}{l}6 \mathrm{~km} \text { (Bay) - S } \\
10 \mathrm{~km}-\mathrm{S}\end{array}$ \\
\hline $\begin{array}{l}\text { Hurricane Jeanne } \\
\qquad(2004)\end{array}$ & KMLB & 3 & 23 & $\begin{array}{l}\text { Suburban- Suburban/ } \\
\text { Marine-Suburban-Airport/ } \\
\text { Suburban }\end{array}$ & $\begin{array}{l}2 \mathrm{~km} \text { (Indian River) - E } \\
6 \mathrm{~km}-\mathrm{E}\end{array}$ \\
\hline $\begin{array}{l}\text { Hurricane Katrina } \\
\text { (2005) }\end{array}$ & KLIX & 3 & 21 & $\begin{array}{l}\text { Forested-Forested-Subur- } \\
\text { ban-Forested }\end{array}$ & $\begin{array}{l}10 \mathrm{~km} \text { (Lake Ponchar- } \\
\text { train) - SSW }\end{array}$ \\
\hline $\begin{array}{l}\text { Hurricane Ophelia } \\
\qquad(2005)\end{array}$ & KMHX & 1 & 24 & $\begin{array}{l}\text { Suburban-Suburban-For- } \\
\text { ested-Suburban }\end{array}$ & $11 \mathrm{~km}-\mathrm{SE}$ \\
\hline $\begin{array}{l}\text { Hurricane Rita } \\
\text { (2005) }\end{array}$ & KBYX & 2 & 27 & $\begin{array}{l}\text { Marine-Marine-Marine- } \\
\text { Marine }\end{array}$ & $\begin{array}{l}\text { VAD domain extends } \\
\text { over water }\end{array}$ \\
\hline $\begin{array}{l}\text { Hurricane Wilma } \\
\text { (2005) }\end{array}$ & $\begin{array}{l}\text { KAMX, } \\
\text { KBYX }\end{array}$ & 3 & $\begin{array}{c}18 \\
(\text { KAMX), } \\
24 \\
(\text { KBYX) }\end{array}$ & $\begin{array}{l}\text { KAMX: Suburban-Subur } \\
\text { ban-Suburban/Farmland- } \\
\text { Suburban }\end{array}$ & KAMX: $10 \mathrm{~km}-\mathrm{E}$ \\
\hline $\begin{array}{l}\text { Hurricane Ike } \\
\text { (2008) }\end{array}$ & KHGX & 2 & 30 & $\begin{array}{l}\text { Suburban-Suburban-Subur- } \\
\text { ban/Farmland-Suburban/ } \\
\text { Farmland }\end{array}$ & 9 km (Trinity Bay) - NE \\
\hline
\end{tabular}
within each $3-5 \mathrm{~km}$ annulus. 
The VAD technique also requires that the horizontal wind field is linear. Therefore the VAD domain was restricted to those data $3-5 \mathrm{~km}$ from the radar site to minimize the influence of flow curvature. The maximum tilt angle was restricted to $14.9^{\circ}$. The raw Doppler velocity data was dealiased and decluttered. Data within the complete volume were binned by height. The assigned heights above ground level (agl) were assumed to be above flat terrain with no significant elevation changes. The assigned height value also included the height of the radar above the ground. For typical scanning strategies, profiles contained 11 bins between 50-1100 $\mathrm{m}$ using all tilt angles below 14.5. An example of a VAD Fourier series fit and derived wind profile is shown in Fig. 3. The size of data contained within each bin was a function of the VCP tilt angle increments. The least-squares Fourier fits were assumed to be representative of the radial velocity field for the corresponding height bin. The obtained wind speed and direction values are a temporal and volumetric average of the Doppler velocity measurements over the time required to complete the volumetric scanning strategy and within each volume of atmosphere assigned to individual height bins. As shown in Table 1, the terrain surrounding most radar sites was typically characterized as rough or suburban. The derived profiles are likely somewhat representative of these general terrain characteristics. It is noted that transitional flow regimes are likely present within the VAD domain. The diameter of the domain is also large enough for the flow to adjust to underlying terrain changes. Therefore, it is unclear if the profile is representative of the effective upstream roughness or if it is simply an average of the roughness azimuthally around the VAD domain.

\subsubsection{VAD quality control and validation}

Quality control procedures were applied to each least-squares Fourier series fit. Individual radial velocity measurements were compared to the initial fit and outliers were identified as data which fell outside twice the mean of the residuals. The outliers were removed and a new fit was analyzed again. Up to five iterations were performed until the fit achieved a coefficient of determination $\left(\mathrm{R}^{2}\right)$ value greater than 0.9. If the value after five iterations did not reach 0.9, the wind speed and direction estimate for the height bin was removed from the profile. The VAD method yielded 383 wind profiles which was comparable to the number of GPS sondes used in previous research (Franklin et al. 2003, Powell et al. 2003, Vickery et al. 2009).

In order to estimate the error associated with the derived wind speed, the residuals from all VAD profiles were examined with respect to the height bin. The distribution of the residuals for each bin

(a)

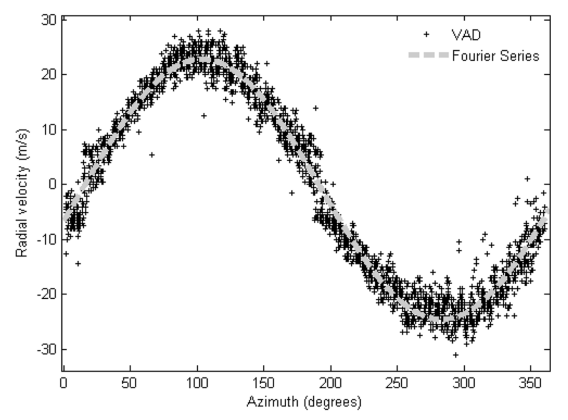

(b)

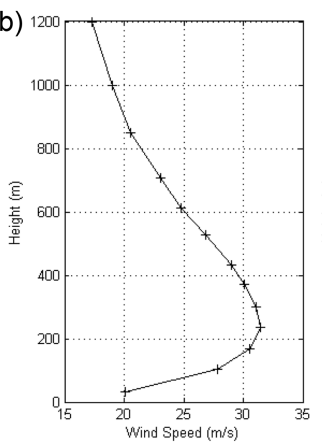

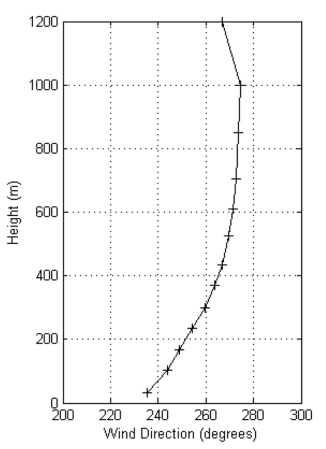

Fig. 3 (a) Velocity azimuth display and Fourier series fit (dashed) for $150 \mathrm{~m}$ height bin and (b) VAD derived wind speed (left) and direction (right) from Hurricane Fran at 0113 UTC 6 September 1996 
was found to be log-normal. The mean of the residuals showed little variation with height and remained near $3 \mathrm{~ms}^{-1}$ as shown in Fig. 4. The standard deviation did increase with height due to the large volume of data included at higher tilt angles. Although this provided a general estimate of the variability within the VAD profile it does not represent a true error estimate. The spread of the velocity data for each height bin included the influence of smaller-scale turbulent features which pass through the VAD domain during the course of each elevation sweep and complete volume (Wurman and Winslow 1998, Lorsolo et al. 2008). The $3 \mathrm{~ms}^{-1}$ value is likely an upper bound of the possible error associated with the wind speed estimate. The precision of the derived winds was limited by the precision of the WSR-88D velocity measurements which is $0.5 \mathrm{~ms}^{-1}$.

Direct measurement comparisons to the VAD profiles were limited to cases in which a GPS sonde was released near a WSR-88D site. The VAD profiles in the four comparisons shown in Fig. 5 were able to capture the general mean structure of the wind profile. Profiles were normalized by the layer mean below $500 \mathrm{~m}$ (Mean Boundary Layer Wind) given the different measurement characteristics of the two platforms. The four GPS sonde profiles shown did not fall within the VAD domain of the individual radar site. It is noted that the GPS sonde data were filtered by an additional 10second low-pass filter in order to remove the influence of coherent turbulent structures, which are likely removed within the VAD technique. The most notable difference amongst the profiles is the steeper slope associated with the VAD profiles. This increase is due to large overland surface roughness lengths. The GPS sonde profiles are representative of an open ocean observation. It is noted that the GPS sonde profiles shown in Fig. 5 were released over water (Lake Ponchartrain for those GPS sondes near the KLIX radar site). The VAD domain associated with the KBYX radar site on Boca Chica Key extended over water; thus the lower portion of the normalized VAD and GPS sonde profiles compare quite well as the two platforms effectively sample the same surface roughness characteristics.

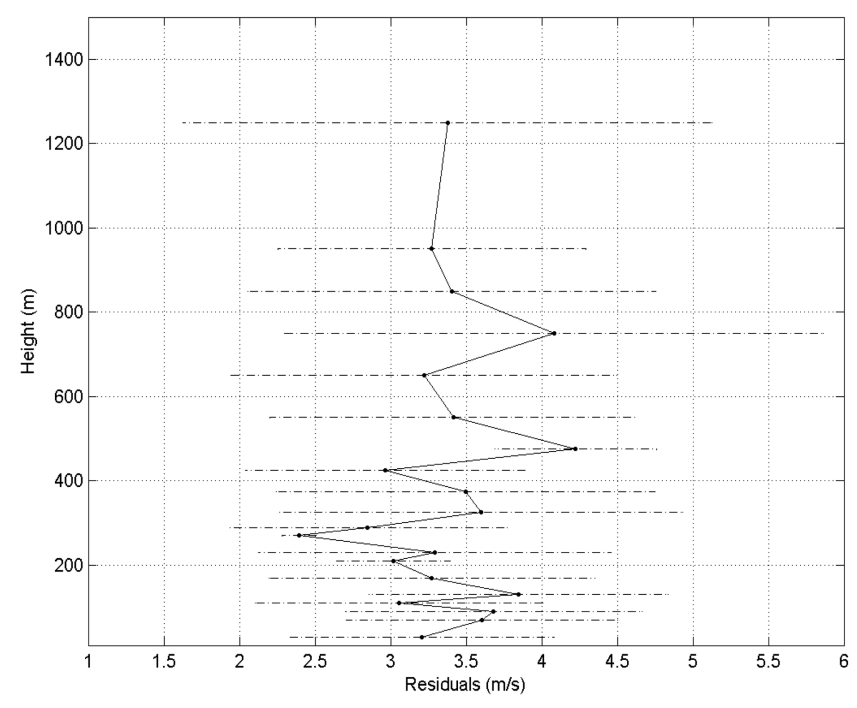

Fig. 4 Mean of the residuals for all VAD profiles binned by height. Horizontal error bar represents \pm 1 standard deviation from the mean 

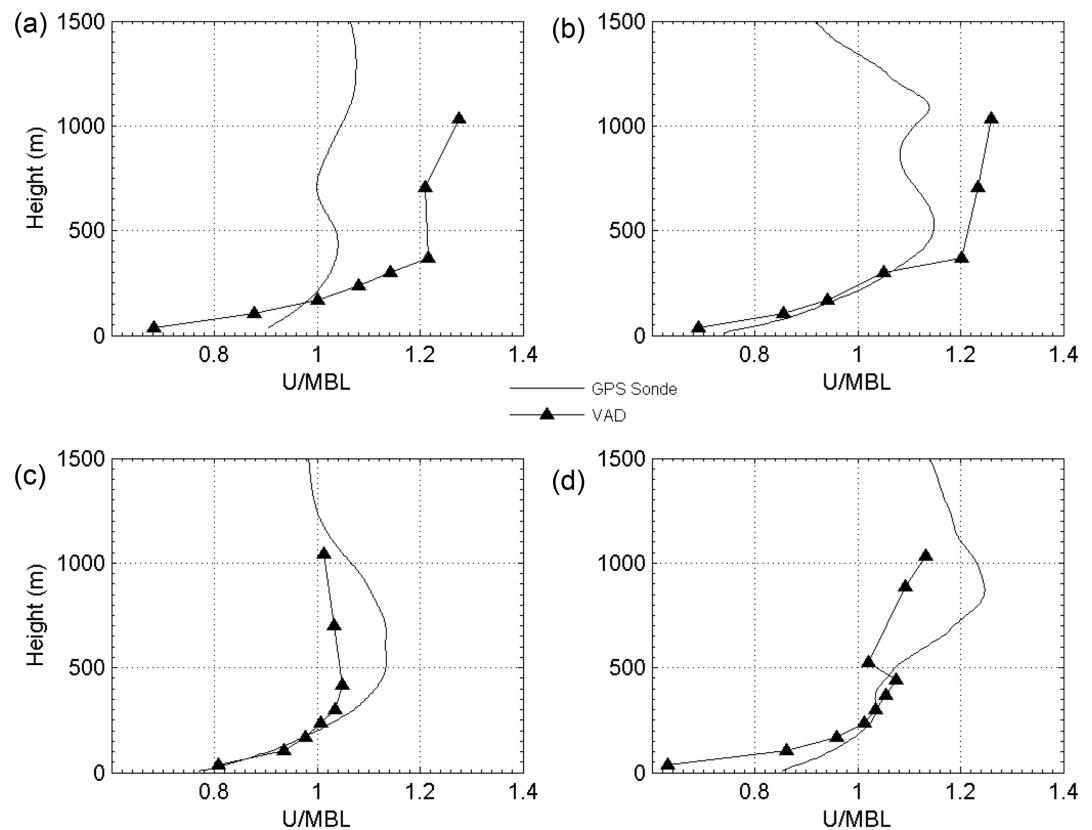

Fig. 5 (a) Normalized KLIX VAD profile from Hurricane Katrina at 1116 UTC 29 August 2005 (triangles) and normalized GPS sonde (solid) launched at 1117 UTC which landed $23 \mathrm{~km}$ to the southwest of the KLIX radar, (b) normalized KLIX VAD profile (triangles) from Hurricane Katrina at 1338 UTC and normalized GPS sonde (solid) launched at 1340 UTC which landed $16 \mathrm{~km}$ to the east-southeast of the KLIX radar, (c) normalized KBYX VAD profile (triangles) from Hurricane Rita at 1821 UTC 20 September 2005 and GPS sonde (solid) launched at 1830 UTC which landed $38 \mathrm{~km}$ to the south of the KBYX radar, and (d) normalized KLTX VAD profile (triangles) from Hurricane Floyd at 0518 UTC 16 September 1999 and normalized GPS sonde (solid) launched at 0514 UTC which landed 16 $\mathrm{km}$ east of the KLTX radar site. and It is noted that an additional 10-s low-pass filter was applied to GPS sonde profiles

\subsection{Measurement characteristics}

The current study employs observing platforms which differ from Eulerian wind measurements. The use of "traditional" anemometer measurements and associated temporal averages (e.g., 3-second gust, 1-minute sustained wind speed) to determine wind flow characteristics and their application toward minimum structural design standards has been common practice within the engineering community as well as within operational meteorology. The use of "non-traditional" observing platforms (e.g., GPS sondes, VAD wind profiles) has yielded a significant amount of information regarding the mean and variance of tropical cyclone vertical wind profiles. Unfortunately, their relationship to typical Eulerian temporal averages is somewhat unknown.

Although the motion of GPS sondes during their descent is well understood and documented; their measurement characteristics with regards to Eulerian observations are not. Measurements made by GPS sondes are collected at $2 \mathrm{~Hz}$ resolution and smoothed using a 5 -second low-pass filter. The observations however are not representative of an Eulerian mean nor are they purely Lagrangian. The instrument package does not follow the motion of individual parcels of air. Angel et al. (1971) provided evidence to suggest that Lagrangian averaging times were typically 3 to 4 times longer 
than Eulerian means for an equivalent horizontal length scale. This suggests that a single GPS sonde processed observation is likely representative of a gust feature smaller than 3 -seconds. However, a sonde likely does not sample the entire length of any given feature as it descends. The profile is subject to any feature which it happens to encounter during the descent. The use of composite profiles, comprised of many GPS sonde measurements, was an effective method to extract a mean representation of the vertical wind profile (Powell et al. 2003, Vickery et al. 2009). The current study employs this method. The generated profiles are representative of an ensemble mean and the influence of convective and smaller scales of motion are buried within the ensemble averages.

Doppler velocity measurements obtained from operational weather surveillance radars (WSR-88D) represent both a temporal and volumetric average. The wind observations are calculated from a large volume of data collected over approximately 8 minutes; the time required for the radar to complete 360 degrees azimuthal rotation for several elevation angles. The VAD technique described in the current study was developed to provide a profile that can be classified as an average, absent of small scale features. All VAD profile wind observations are a volumetric average. Similarly to GPS sondes, the relationship between a VAD wind speed using the prescribed method and a conventional Eulerian observation is unknown. A single comparison was made using a Texas Tech University $10 \mathrm{~m}$ tower deployed within the VAD domain of the KLIX WSR-88D radar during the landfall of Hurricane Katrina (2005). A logarithmic fit was applied to the lowest $300 \mathrm{~m}$ of the VAD profile and used to estimate the $10 \mathrm{~m}$ wind speed. Tower measurements were segmented using a variety of averaging times centered on the time of each VAD profile for the selected period of observation. The use of a 10-minute mean resulted in the smallest mean error of $-1.2 \mathrm{~ms}^{-1}$ and the 10-minute mean tower measurements throughout the time series remained within the estimated VAD error of $\pm 3 \mathrm{~ms}^{-1}$. The preliminary result suggested a VAD wind measurement may be similar to a 10-minute mean from a conventional propeller-vane anemometer. It is noted that this represents a single comparison and additional comparisons are sorely needed to determine if this relationship is valid. The result is promising and indicates that a VAD profile maybe used to estimate a $10 \mathrm{~m}$ wind speed through the application of surface layer similarity theory.

\section{Methodology}

Composite profiles were produced for both GPS sonde and VAD wind profiles. The mean boundary layer wind (MBL) was selected to normalize the wind observations according to Powell et al. (2003). The MBL wind speed is the mean of GPS sonde wind speed observations from the lowest $500 \mathrm{~m}$ of the profile. The layer average typically includes boundary layer observations and the wind speed maximum near the top of the HBL. The use of the MBL to normalize wind measurements also mitigates influences on the profile from the outward tilt of the radius of maximum winds with height above the boundary layer (Jorgensen et al. 1984b, Powell et al. 2009). GPS sonde data were also grouped by MBL using $5 \mathrm{~ms}^{-1}$ bin sizes. Individual sonde observations were binned by height according to Powell et al. (2003). Composite profiles were produced for each MBL wind group assuming that each individual observation represented a perturbation from a mean state for the given group. Both GPS sonde and VAD profiles were also stratified by radial distance from the cyclone center and storm-relative azimuth. Normalized profiles were computed for each group. 


\section{Characteristics of tropical cyclone vertical wind profiles}

\subsection{Over water mean wind profile characteristics}

A composite profile was generated from all 1080 GPS sonde profiles, shown in Fig. 6. Nearly 430,000 observations were used to produce the composite which contained a broad wind speed maximum near $625 \mathrm{~m}$ and a logarithmic increase in wind speed from the surface to the wind maximum $\left(\mathrm{U}_{\max }\right)$. The height of the maximum $\left(\mathrm{z}_{\max }\right)$ was slightly higher than the mean profiles of Powell et al. (2003) and Franklin et al. (2003). This was likely due to the inclusion of a larger number of GPS sondes from all regions of tropical cyclones as both studies used primarily sondes released within the eyewall. Beneath the wind maximum, the decrease in standard deviation with height was indicative of a general decrease in turbulence intensity with height. The result was anticipated given the often assumed neutral stability of the hurricane boundary layer.

The composite profiles for each MBL wind speed group indicate a general decrease in the height of the wind maximum with increasing MBL wind speed. The height of the radial inflow layer also decreased slightly with increasing MBL, but remained above the wind maximum as shown in Fig. 7. The result argued that the HBL decreases in depth as the mean winds increase. The decrease in surface roughness shown by Powell et al. (2003) as surface wind speeds increase above $40 \mathrm{~ms}^{-1}$ support the reduction in boundary layer depth. Interestingly, the GPS sonde composite profile revealed the presence of a constant inflow layer that was typically $150 \mathrm{~m}$ deep. The layer depth did not show a dependence upon the mean wind speed as shown in Fig. 7. Composite profiles from the radial groupings also revealed a decrease in the height of the wind maximum with decreasing radius, as shown in Fig. 8. The characteristics of the underlying sonde profiles also revealed a

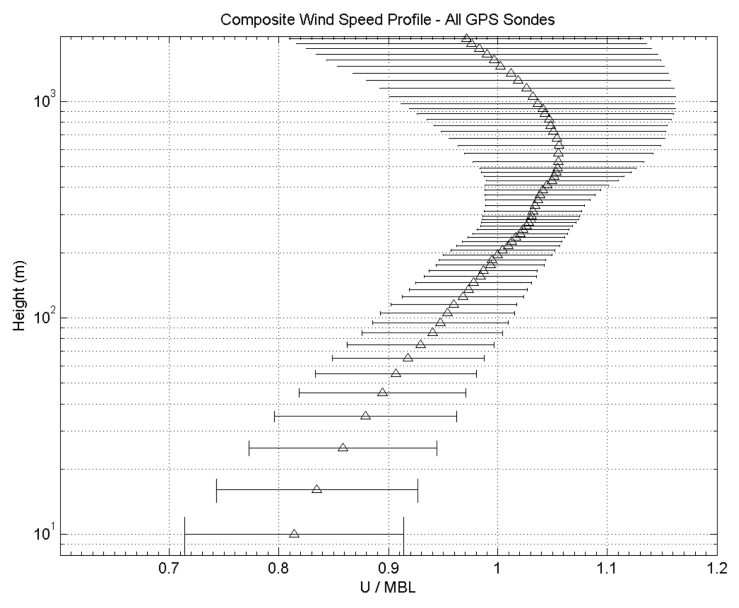

Fig. 6 Normalized composite wind speed (U) profile for all GPS sondes. Error bars represent \pm 1 standard deviation from the mean for each height bin. Values represent an ensemble mean

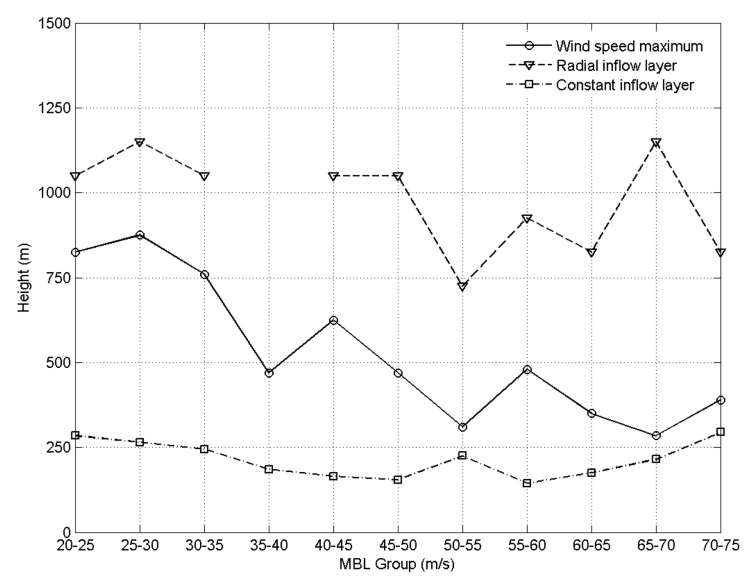

Fig. 7 Height of the wind maximum, radial inflow layer, and constant inflow layer shown as a function of $\mathrm{MBL}$ wind group. The inflow layer group for the $35-40 \mathrm{~ms}^{-1}$ group was a significant outlier with a height of $2 \mathrm{~km}$ and was excluded

similar relationship, shown in Fig. 9. The peak wind speed in each profile decreased in height as 


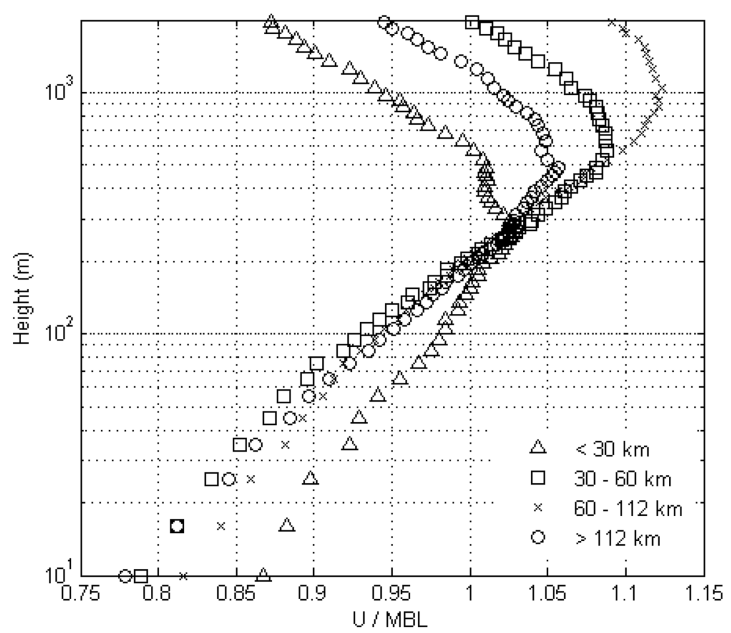

Fig. 8 Normalized composite wind speed (U) profile Fig. for radial GPS sondes groups. Values represent an ensemble average

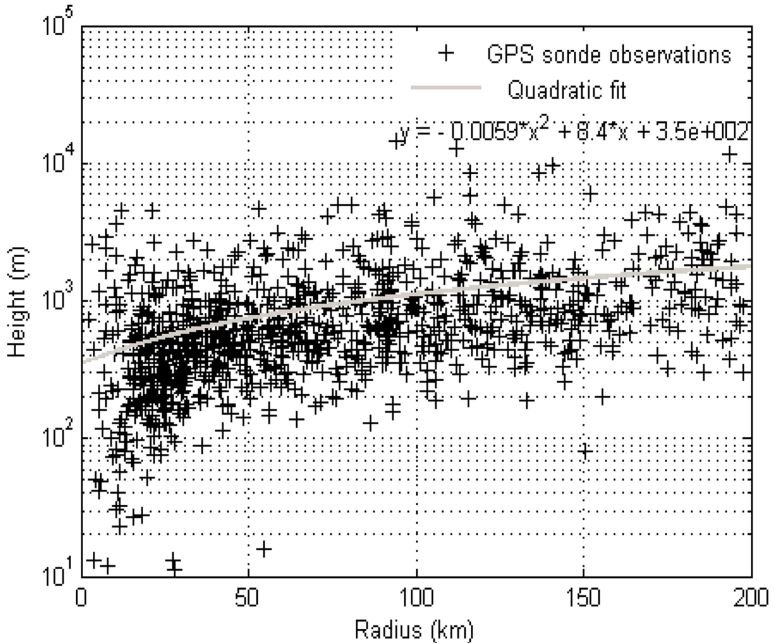

9 Absolute wind speed maxima observed by GPS sondes shown as a function of splash radius. The least-squares quadratic fit is shown in gray

radial distance decreased. Maxima below $200 \mathrm{~m}$ were typically confined to radii less than $60 \mathrm{~km}$. The scaled magnitude of individual wind maxima also decreased with decreasing radial distance from the storm center, as shown in Fig. 10. Local wind maxima within GPS sonde profiles also followed a similar relationship as larger perturbations from the MBL wind were found at large radii. While at small radii, likely near the eyewall, departures from the layer mean were typically less than $10 \%$.

The result was strikingly similar to that found by Schroeder et al. (2009) who examined over-land surface gust factors as a function of radius. For a specific roughness regime larger gust factors were observed within rainband regions at large radii. It is hypothesized that the wind maximum at the top

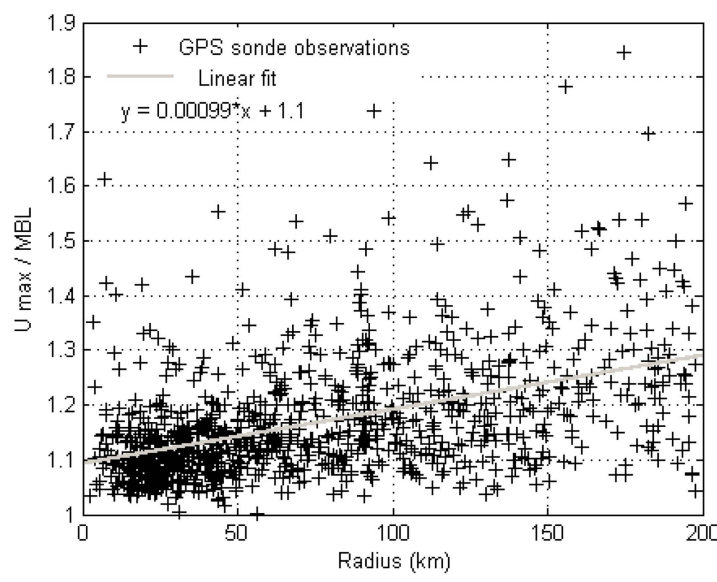

Fig. 10 Scaled peak wind speed for all GPS sondes shown as a function of splash radius. The least-squares linear fit is shown in gray

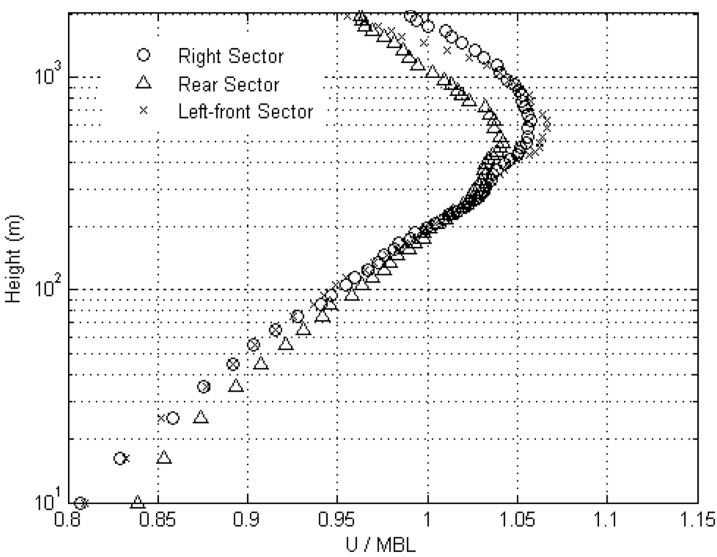

Fig. 11 Normalized composite wind speed profile for storm-relative azimuth angle GPS sondes groups. Values represent an ensemble average 
of the boundary layer is a reasonable estimate of the upper bound of anticipated maximum surface wind gusts. At small radii near the eyewall and surface radius of maximum winds (RMW), the scaled momentum available for vertical transport to the surface is not significantly larger than the layer mean flow. This may provide an explanation for the rarity of extreme gusts (gust factor $>2.0$ ) within the eyewall region. However, at large radii within the rainband region the wind maximum aloft is often substantially greater than the layer mean flow. Therefore larger surface gust factors would be expected at larger radii. Differences between the convective nature of the two regions may have contributed to the observed relationship.

Recent studies have suggested azimuthal variability in the vertical wind profile as well as the underlying wave field in open ocean conditions (Kepert 2006a,b, Moon et al. 2004, Powell 2007, Schwendike and Kepert 2008, Powell et al. 2009). The azimuthal variability in roughness has been hypothesized to be a result of the relative age of the underlying wave field. Moon et al. (2004) has provided evidence that taller more developed waves located in the right sector lead to higher roughness values. The rear and left-front sectors were characterized by smaller and younger wave fields. The results of these studies provided motivation to stratify GPS sondes by storm-relative azimuthal sectors. Storms were divided into three azimuthal sectors: Rear sector (151-240); Right sector (21-150); Left-front (241-20) according to wave characteristics summarized by Black et al. (2007) and based on Wright et al. (2001).

Normalized mean profiles were computed for each storm-relative sector and are shown in Fig. 11. The height of the wind speed maximum was near $600 \mathrm{~m}$ for the right and left-front sectors, while the rear sector exhibited a maximum at $400 \mathrm{~m}$. The left-front scaled wind maximum produced the largest deviation from the MBL at 7\%. The lower wind maximum height noted in the rear sector correlated well with the smoother roughness regime at higher wind speeds within this sector noted by the preliminary work of Powell (2007).

\subsubsection{Log and power law profile comparisons}

Logarithmic and power law wind profiles were examined with respect to GPS sonde composite profiles for each MBL wind speed group. The use of logarithmic and power law wind profiles to estimate the underlying mean wind profile given a constant roughness or shear exponent $(\alpha)$ are contained within the minimum design standards (ASCE 7-10). Powell et al. (2003) and Vickery et al. (2009) have shown that a typical surface layer remains logarithmic as the mean wind speed increases. It was also shown by Vickery et al. (2009) that a logarithmic increase in wind speed extends to the height of the mean wind speed maximum. Logarithmic and power law least-square fits were performed on the composite profiles in order to test the validity of both methods in estimating the surface layer wind profile as well as to validate the historical work of Powell et al. (2003) and Vickery et al. (2009) using a much large dataset. The logarithmic wind profile is typically given in the form

$$
\bar{U}=\frac{u_{*}}{k} \ln \left(\frac{z}{z_{o}}\right)
$$

where $\bar{U}$ represents the mean wind speed, $u_{*}$ is the friction velocity, which is a measure of shear stress, $k$ is the von Karman constant and often assumed to have a value of $0.4, z$ represents height, and $z_{o}$ is the aerodynamic roughness length (Stull 1988). The most common version of the powerlaw is shown by 


$$
\frac{\bar{U}_{z}}{U_{r}}=\left(\frac{z}{z_{r}}\right)^{\alpha}
$$

where $\bar{U}_{z}$ is the mean wind speed at a given height $z ; U_{r}$ is a known wind speed at height $z_{r}$, and $\alpha$ represent an exponent which describes the vertical shear for a given atmospheric stability. Given neutral stability conditions, $\alpha$ is assumed to be $1 / 7$. The use of a constant exponent does not account for surface roughness, displacement height, or changes in atmospheric stability. Hsu et al. (1994) have shown the power law's applicability over the ocean in neutral stability conditions, while Amano et al. (1999) have applied it to the HBL.

Least-squares regressions were applied to a surface layer of 20-160 m AGL as this layer is representative of a layer depth in which the wind direction was typically constant. The failure of GPS sondes to transmit data increases significantly below $20 \mathrm{~m}$ (Powell et al. 2003). The inclusion of observations at and above $20 \mathrm{~m}$ enabled a larger amount of data to be contained within the mean statistic for the lowest bin relative to including the $10 \mathrm{~m}$ bin. Both the power and logarithmic fits performed quite well as shown in Fig. 12, in agreement with previous studies (Powell et al. 2003, Vickery et al. 2009). As shown in Table 2, the log-law performed slightly better, with an average $\mathrm{R}^{2}$ value 3\% higher than the power-law least-squares fit. Although values of the shear exponent were smaller than the typical standard $1 / 7$ value, smaller surface roughness values in open ocean
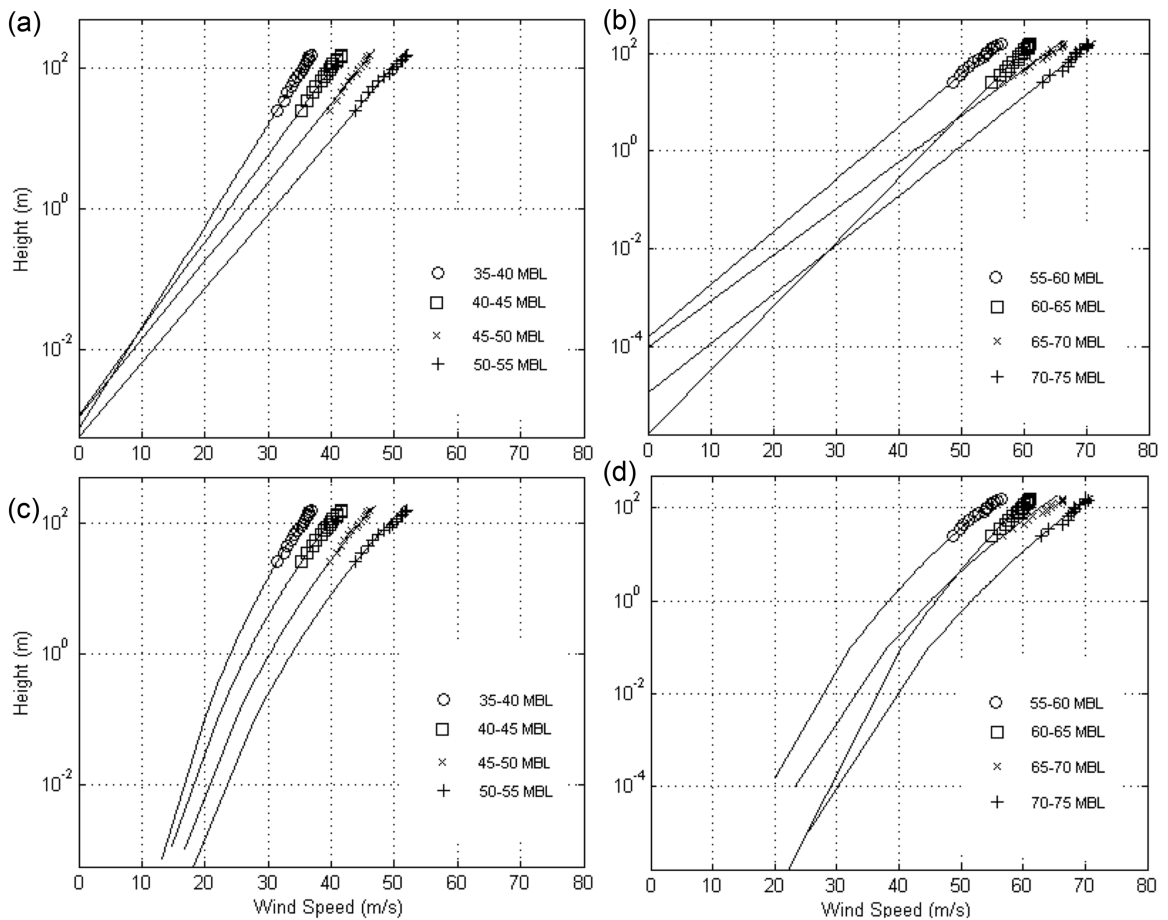

Fig. 12 (a) Logarithmic least-squares fits for 35-40, 40-45, 45-50, 50-55 $\mathrm{ms}^{-1} \mathrm{MBL}$ wind groups, (b) logarithmic least-squares fits for 55-60, 60-65, 65-70, 70-75 $\mathrm{ms}^{-1} \mathrm{MBL}$ wind groups, (c) exponential (Power Law) least-squares fit for 35-40, 40-45, 45-50, 50-55 $\mathrm{ms}^{-1} \mathrm{MBL}$ wind groups and (d) exponential (Power Law) least-squares fit for 55-60, 60-65, 65-70, 70-75 $\mathrm{ms}^{-1} \mathrm{MBL}$ wind groups. All fits are for a 20-160 m surface layer 
Table 2. Results of log and power law least-squares fits for a $20-160 \mathrm{~m}$ surface layer.

\begin{tabular}{cccccc}
\hline \hline MBL group $\left(\mathrm{ms}^{-1}\right)$ & $\begin{array}{c}\text { Surface layer } \\
\text { depth }(\mathrm{m})\end{array}$ & $\begin{array}{c}25 \mathrm{~m} \text { bin sample } \\
\text { size }\end{array}$ & $R^{2}$-Log & $R^{2}$-Power & $\alpha$ \\
\hline $35-39.999$ & $20-160$ & 236 & 0.996 & 0.993 & 0.086 \\
$40-44.999$ & $20-160$ & 210 & 0.994 & 0.991 & 0.093 \\
$45-49.999$ & $20-160$ & 166 & 0.993 & 0.992 & 0.083 \\
$50-54.999$ & $20-160$ & 103 & 0.989 & 0.952 & 0.098 \\
$54-59.999$ & $20-160$ & 105 & 0.989 & 0.968 & 0.081 \\
$60-64.999$ & $20-160$ & 54 & 0.992 & 0.975 & 0.058 \\
$65-69.999$ & $20-160$ & 64 & 0.992 & 0.991 & 0.089 \\
$70-74.999$ & $20-160$ & 53 & 0.962 & 0.941 & 0.061 \\
\hline
\end{tabular}

conditions resulted in the value. The vertical extent of the layer in which the least-squares regressions were performed was incrementally increased to the height of the mean wind speed maximum and the fits were recalculated. For both the $\log$ and power law profiles, $\mathrm{R}^{2}$ values did not fall below 0.9 through the depth of the maximum. The performance of the least-squares fits for each method validated their use in representing the surface layer wind speed profile in open ocean conditions given suitable estimates of $z_{\mathrm{o}}$ or $\alpha$.

\subsection{Landfall vertical wind profiles}

The VAD wind profiles were examined in a similar fashion to the GPS sonde dataset. A composite profile was generated from all VAD profiles. The composite profile showed a logarithmic increase in wind speed through the lowest $400 \mathrm{~m}$ of the profile, as shown in Fig. 13. The wind speed increased through the depth of the profile and the maximum was located at the vertical extent of the profile. The more rapid increase in wind speed with height within the lowest $400 \mathrm{~m}$ was indicative of a larger effective roughness length when compared to GPS sonde composite profiles
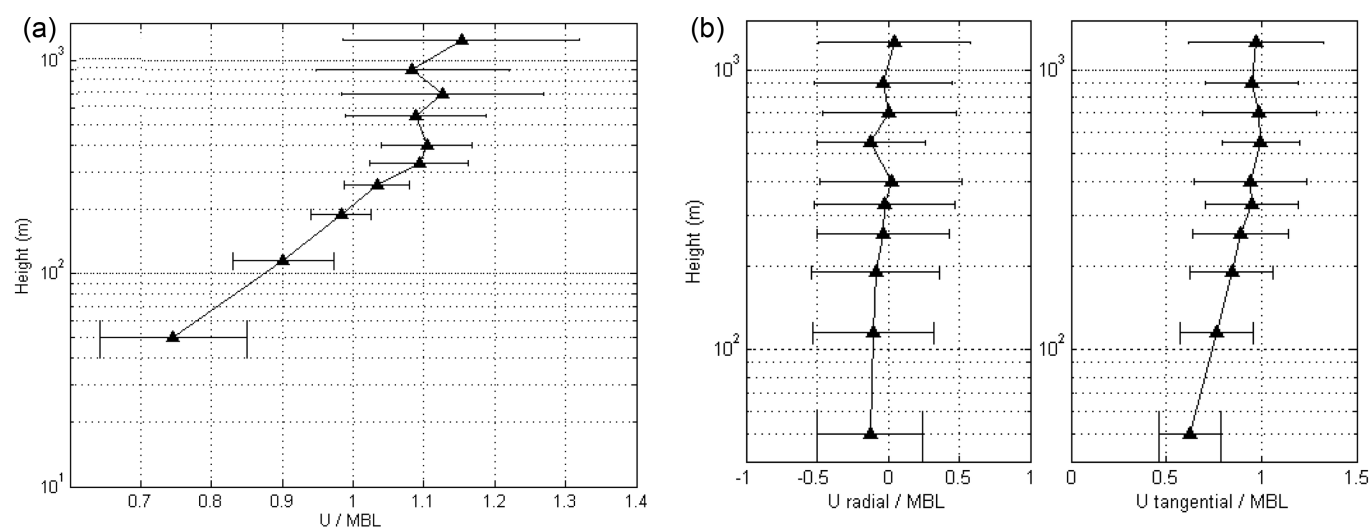

Fig. 13 (a) Normalized wind speed composite VAD wind profile and (b) normalized composite VAD radial (left) and tangential (right) wind profile. Error bars represent \pm 1 standard deviation from the mean for each height bin 
over open ocean conditions (Powell et al. 2003). The result was anticipated given the larger roughness lengths typically found within the VAD domain (Table 1). The Key West (KBYX) radar site was the only site in which the terrain exposure was azimuthally homogeneous and representative of marine exposure characteristics. The radial wind profile contained a maximum at the lowest height bin and decreased with height. Above $500 \mathrm{~m}$ the radial wind component approached zero. The variability and large standard deviations above $300 \mathrm{~m}$ were likely a result of internal boundary layer (IBL) characteristics as well as storm-scale variability. However, the general decrease in standard deviation values with height below $300 \mathrm{~m}$ supported a decrease in turbulence intensity, similar to that shown by GPS sonde composite profiles over water and indicative of neutral stratification.

Stratification by radius yielded similar results to the GPS sonde composites. A jet-like wind maximum was only apparent for the inner-most group as shown in Fig. 14. The remainder of the profiles exhibited a well-mixed boundary layer, with a wind speed increase throughout the depth of
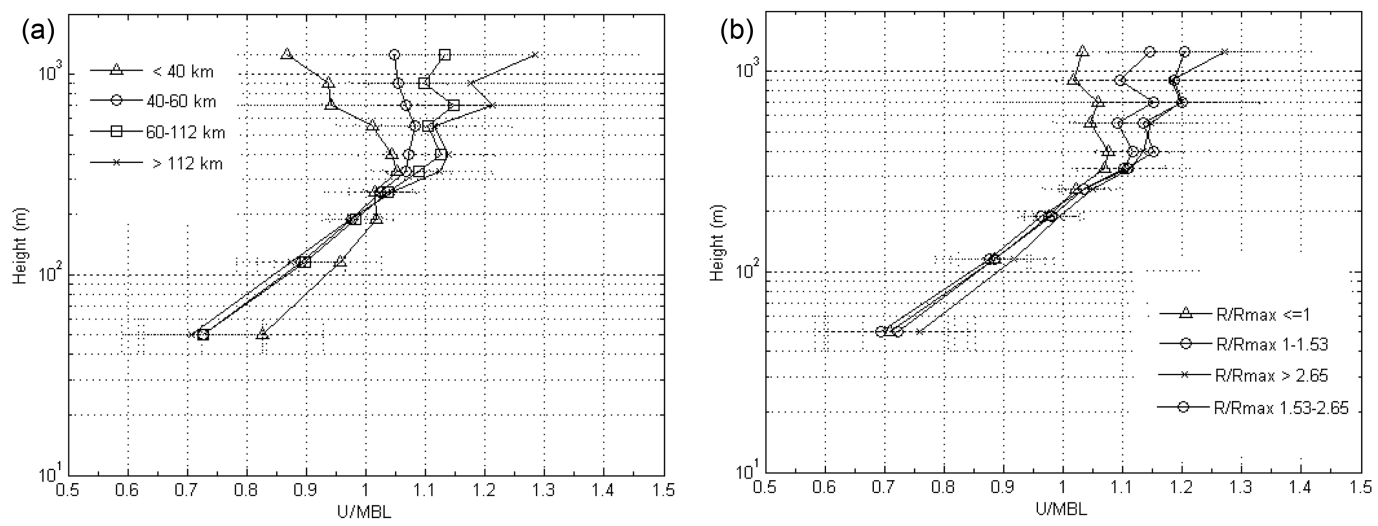

Fig. 14 (a) Normalized composite VAD wind speed profiles for radial groups and (b) normalized composite VAD wind speed profiles for scaled radial groups. Error bars (dashed) represent \pm 1 standard deviation from the mean for each height bin
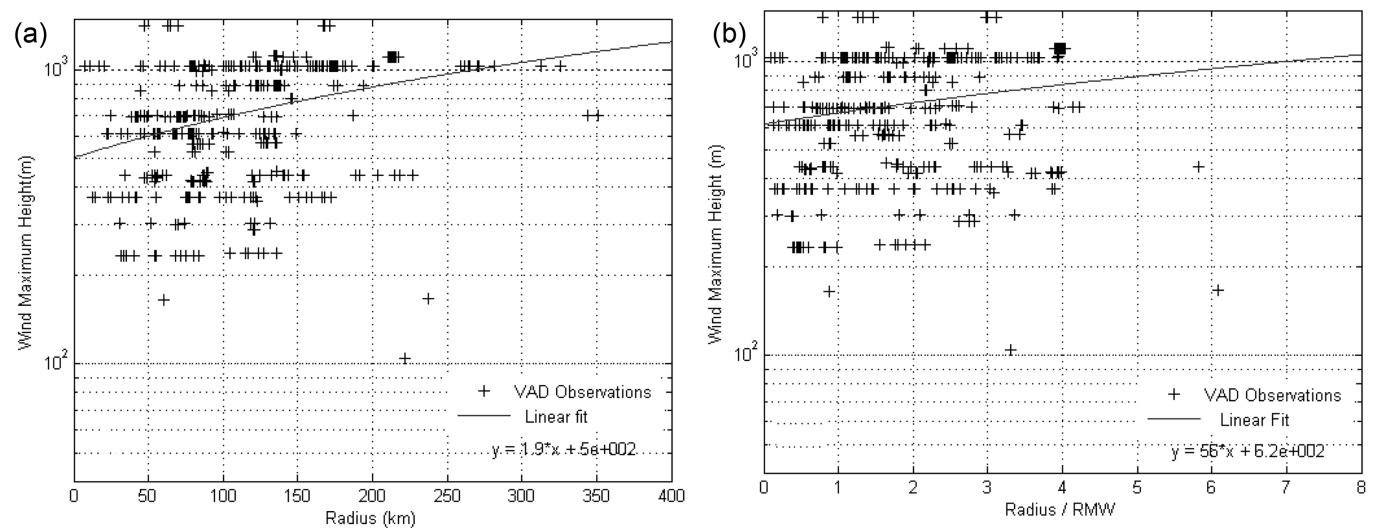

Fig. 15 (a) Height of the VAD observed wind maximum shown as a function of radius and (b) height of the VAD observed wind maximum shown as a function of scaled radius. The linear least-squares fits are shown by the solid black line 
the profile. The underlying VAD profiles exhibited a general trend toward higher wind maximum heights at large radii shown in Fig. 15, but the relationship was not as pronounced as that exhibited by GPS sonde observations. The reduction in the correlation may be due to the relative coarse resolution of the VAD profiles as compared to GPS sondes. The scaled magnitude of the wind maximum increased greatly with radius similar to that observed by GPS sondes. Fig. 16 illustrates the strong correlation between the scaled magnitude of the maximum and radial distance.

\subsubsection{Log and power law comparisons}

The performance of $\log$ and power law wind profiles was also quantified for the VAD wind profiles for a 50-400 $\mathrm{m}$ layer or through the height of the wind speed maximum if it was found below $400 \mathrm{~m}$. The large depth was selected in order to increase the number of observations included in the least-squares fit which typically contained 5-7 observations for most radar VCP. The mean $\mathrm{R}^{2}$ values for the $\log$ and power law least-squares fit were 0.93 and 0.86 respectively. The log law performed the best with a mean error of $0.013 \mathrm{~ms}^{-1}$ whereas the power law exhibited a mean error of $0.35 \mathrm{~ms}^{-1}$. The log law generally underestimated the wind speed in the lowest height bin. The result is likely due to the influence of IBL processes and lack of observations below $50 \mathrm{~m}$. Given the resolution of the VAD profiles, any IBL development or growth will likely be buried within the Fourier series fit for the specific height bin. Additionally, the wind maximum aloft may help to increase the slope of the profile such that the least-squares fit cannot capture the true wind speed at the lowest bin. For the remaining bins, the mean errors associated with the logarithmic fit were very near zero with a slightly larger spread above $200 \mathrm{~m}$. The power law exponent $(\alpha)$ was found to have a mean value of 0.18 which is slightly larger than the $1 / 7$ value. Given the general rough terrain characteristics surrounding most radar sites, the result was expected as the $1 / 7$ value applies to open terrain exposure. Values of $\alpha$ also decreased as the MBL increased as shown in Fig. 17 as the slope of the profile changed.

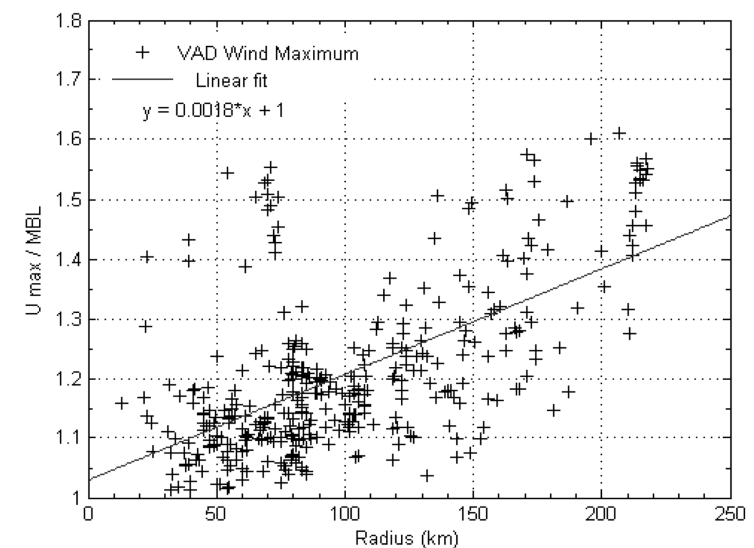

Fig. 16 Scaled VAD observed wind speed maximum shown as a function of radius. The linear least-squares fit is shown by the solid black line

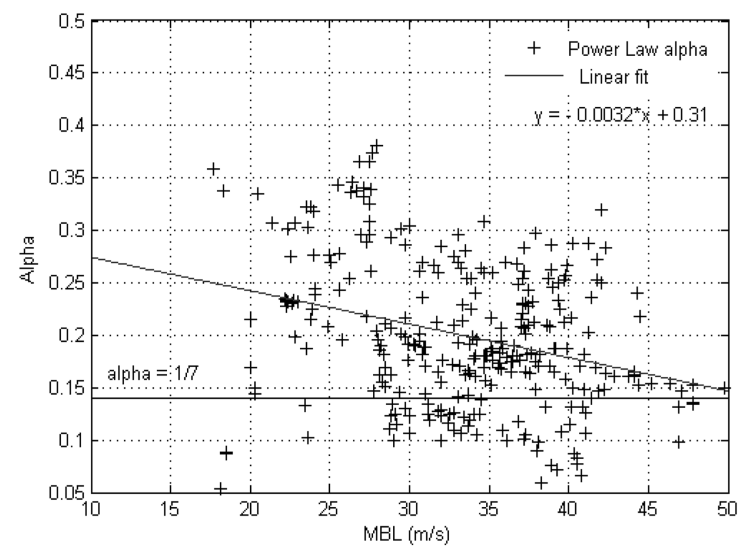

Fig. 17 Shear exponent $(\alpha)$ from the least-squares exponential fit for VAD profiles shown as a function of MBL wind speed. 1/7 values is shown by the solid black line. The leastsquares linear fit is also shown in black 


\subsubsection{Evolution of vertical wind profiles}

The modified VAD technique provided information regarding the temporal and spatial evolution of the vertical wind profile. The surface radius of maximum wind (RMW) was estimated from surface observations using the $\mathrm{H}^{*}$ Wind wind field analyses model for the quadrant in which the radar site was located (Powell et al. 1998). A cubic spline was fit to the time history of RMW values in order to describe the temporal evolution of the RMW. Time series of vertical wind profiles were developed for each observation period of interest, and jet-like wind profiles were identified. The features were defined as a clear wind speed maximum within the vertical depth of the wind profile with a wind speed decrease of at least $3 \mathrm{~ms}^{-1}$ (estimated VAD error) from the wind maximum to the lowest height bin and from the wind maximum to the top of the profile similar to Bonner (1968). A summary table of identified jet structures is provided in Table 3.

Several interesting characteristics were noted when the evolution of the vertical wind profile was examined. A general descent was often observed in the wind maximum height with time and decreasing radial distance. The wind maximum typically reached its lowest altitude radially inward from the surface radius of maximum winds for cases in which the surface RMW passed the VAD domain. Profiles often transitioned from a well-mixed shape with an increase in wind speed through

Table 3 Summary of all VAD identified jet features. It is noted that all VAD profiles from Hurricane Bertha and Hurricane Ophelia contained a jet feature. No jet features were identified within the Hurricane Katrina VAD observation domain

\begin{tabular}{|c|c|c|c|c|c|c|c|}
\hline Storm & Radar & Time (UTC) & $\begin{array}{l}\text { Mean height } \\
\text { (m) }\end{array}$ & $\begin{array}{l}\text { Mean scaled } \\
\text { maximum }\end{array}$ & $\begin{array}{c}\text { Storm relative } \\
\text { sector }\end{array}$ & Radius (km) & Region \\
\hline Bertha & KLTX & $1406-1754$ & 594 & 1.25 & Left-front & $74-45$ & Outer-vortex \\
\hline Fran & KLTX & $0108-0349$ & 434 & 1.18 & $\begin{array}{l}\text { Left-front } \\
\text { to Rear }\end{array}$ & $103-25$ & Eyewall \\
\hline Bonnie & KLTX & 1604-1609 & 442 & 1.10 & Left-front & 143 & Outer-vortex \\
\hline Dennis & КМHX & $1704-1808$ & 743 & 1.45 & Left-front & $107-100$ & \\
\hline Georges & KBYX & 1249 & 238 & 1.06 & Left-front & 104 & Outer-vortex \\
\hline Georges & KBYX & $1359-1524$ & 517 & 1.20 & Left-front & $58-22$ & Eyewall \\
\hline Georges & KBYX & $1644-1840$ & 303 & 1.04 & Right & $53-75$ & $\begin{array}{l}\text { Eyewall - } \\
\text { outer vortex }\end{array}$ \\
\hline Isabel & КMHX & 1059 & 612 & 1.23 & Left-front & 128 & Outer-vortex \\
\hline Isabel & KMHX & $1639-1913$ & 479 & 1.08 & Left-front & $93-13$ & Eyewall \\
\hline Frances & KMLB & $0422-0634$ & 583 & 1.16 & Right & $127-122$ & Outer-vortex \\
\hline Jeanne & KMLB & 0026 & 416 & 1.10 & Right & 162 & Outer-vortex \\
\hline Jeanne & KMLB & $0822-0843$ & 696 & 1.06 & Right & $79-75$ & Outer-vortex \\
\hline Katrina & KLIX & NA & NA & NA & NA & NA & NA \\
\hline Ophelia & KMHX & $2025-2355$ & 627 & 1.20 & Left-front & $93-69$ & Eyewall \\
\hline Rita & KBYX & 1904 & 420 & 1.25 & Right & 83 & Outer-vortex \\
\hline Wilma & KBYX & 0846 & 371 & 1.27 & Left-front & 106 & Outer-vortex \\
\hline Wilma & KAMX & 1316-1354 & 705 & 1.18 & Right & $83-107$ & Outer-vortex \\
\hline Ike & KHGX & $0607-0718$ & 437 & 1.15 & Left-front & $60-39$ & Eyewall \\
\hline
\end{tabular}




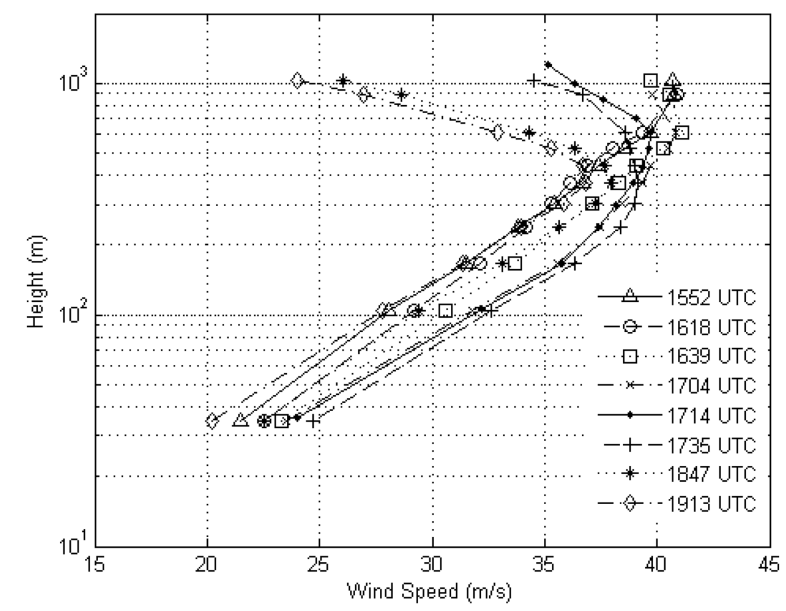

Fig. 18 KLTX VAD profiles from Hurricane Floyd between 1550 and 1920 UTC on 16 September 1999

the depth toward the presence of a jet feature as shown in the example in Fig. 18 from Hurricane Floyd (1999). As shown in Table 3, prolonged jet structures were often present to the left of the cyclone track within the off-shore flow regime. Knupp et al. (2006) has provided some observational evidence of jet structures within the off-shore flow regime and Schwendike and Kepert (2008) suggested that this regime would be favorable for jet features due to the acceleration of the tangential flow above the surface near the land-sea interface. Events included in the VAD analysis which the surface RMW passed the radar site, were confined to the off-shore flow regime. It is unclear if a similar structure and evolution occurs within the on-shore flow regime. This highlights the continued need for additional analysis as more data becomes available.

Although the wind profile typically exhibited a well-mixed shape at large radii, the passage of convective elements within rainband features induced changes to the wind profile. In some instances short-lived jet structures were observed as a wind maximum developed near $500 \mathrm{~m}$, as shown in Table 3. Following the passage of the band, the profile returned to a well-mixed shape. The shortlived features were typically found to the right of the cyclone track. The increase in the magnitude of the wind maximum aloft was often not reflected in the lowest bin of the VAD profile which allowed for larger perturbations from the MBL aloft. This may provide a mechanism which contributes to the larger gust-factors observed at larger radii within roughness regimes shown by Schroeder et al. (2009). The momentum available for vertical transport from aloft is increased due to the presence of the jet feature associated with the band, while the surface mean wind field exhibits little change. Kepert and Wang (2001) hypothesized that any region with enhanced updrafts, such as a rainband, may be a favored location for boundary layer jet development through increased inward advection of angular momentum and the vertical transport of radial momentum through convective updrafts. The jet described by Kepert and Wang (2001) represented a departure from gradient balance, unfortunately it is difficult observationally to measure the gradient wind and a direct comparison cannot be made (Willoughby 1990). 


\section{Conclusions}

The deployment of GPS dropwindsondes into the hurricane environment has provided a wealth of information regarding the kinematic and thermodynamic structure of the HBL. Over 1000 GPS sondes were employed to examine the characteristics of tropical cyclone vertical wind profiles. The generation of composite wind profiles for various MBL wind groups and radial and storm-relative stratifications provided information regarding the mean wind maximum found at the top of the hurricane boundary layer. The use of a modified VAD wind profile technique proved to be effective in mitigating the data void typically found at landfall. Over 300 VAD profiles were used to evaluate the characteristics of tropical cyclone wind profiles at landfall and their evolution.

The examination of composite GPS sonde profiles revealed a reduction in boundary layer depth as the mean wind state of the environment increases. The composite technique removes the influence of convective motions and is an effective representation of the wind maximum near the top of the boundary layer. The reduction of the mean inflow layer depth also supported the compression of the boundary layer as mean wind speed increase toward the cyclone center. Although the wind maximum is reduced in height, the profile beneath remained logarithmic. Individual profiles also supported this relationship as their statistics are tied to the underlying mean profiles. When normalized by the MBL wind, the scaled wind maximum decreased in magnitude with increasing MBL wind speed. Although a lower wind maximum would be included within the MBL layer average, a more peaked maximum would still result in a higher scaled value. The result can be tied to the underlying decrease in roughness as mean winds increases above $40 \mathrm{~ms}^{-1}$ (Powell et al. 2003). This allows the lower portion of the profile to increase in scaled magnitude while the wind maximum aloft remains unchanged.

The decrease in the height of the wind maximum was also reflected in the radial stratification as the two are somewhat linked. Kepert (2001) has provided theoretical evidence to support a radial dependence governed by the shape of the gradient wind profile. Composite profiles revealed a distinct descent of the wind maximum moving inward toward the cyclone center and were supported by the characteristics of the absolute wind maxima from GPS sondes. Also noted was the decrease in the scaled maximum in both composite and individual GPS sonde statistics. The same relation was found at landfall within the VAD derived wind profiles. Given the markedly different roughness conditions sampled by GPS sondes compared to VAD profiles, it appears that the radial descent of the wind maximum is not entirely governed by surface roughness effects and is likely a result of the mechanisms described by Kepert (2001). Within these dynamical processes, surface roughness is a contributing factor due to the dependence in surface drag coefficient and turbulent diffusivity. The relative magnitude of the wind maximum also decreased with decreasing radius and there was some evidence to suggest that lower wind maxima coincided with strong surface tangential winds, but the relative magnitude of the maxima was reduced. Kepert (2001) and Kepert and Wang (2001) provided theoretical evidence to support a stronger wind maximum in regions of strong radial inflow, owing to the contribution of the inward advection of angular momentum. Thus within the eyewall region where the flow becomes more tangential, the jet height is reduced along with the relative magnitude.

The use of VAD derived wind profiles also provided the opportunity to evaluate the evolution of the wind profile at landfall. It was found that the passage of convective elements, often associated with rainband structures, produced a perturbation aloft in the wind profile. This perturbation resulted in short-lived jet structures. Prolonged jet structures were primarily identified within the offshore 
flow regime left of the cyclone track. The associated wind maximum often reached its lowest altitude radially inward from the surface RMW. Limited observational and theoretical studies suggest that the offshore flow regime would likely support a lower jet feature due to the acceleration of the tangential flow as the underlying surface transitions from land surface to water (Knupp et al. 2006, Schwendike and Kepert 2008). Unfortunately, the employed dataset lacks sufficient profiles from the onshore flow regime to make any significant conclusions.

The quality of power law and logarithmic fits to the composite profiles supports the use of these techniques within structural design standards to represent the mean wind profile. The applicability of these relationships is dependent upon a suitable estimate of the surface roughness length (logarithmic law) or the shear exponent (power law). The associated gust envelope for the specified roughness regime would also be considered appropriate. The results of the current study and those of Schroeder et al. (2009) suggest that near-surface gust characteristics may be influenced by the evolution of the vertical wind profile and hurricane dynamical processes. The radial and azimuthal asymmetries noted in composite vertical wind profiles possibly influence the near-surface gust characteristics. At large radii, the structural design standards may be met or exceeded. Given the relative weak mean wind found at large radii, the presence of peaked wind maxima aloft and large near-surface gust factors do not appear to be a design concern. Near the radius of maximum winds, the boundary layer is compressed and the height of the wind maximum approaches $300 \mathrm{~m}$. The scaled wind maximum aloft is also reduced in magnitude suggesting a reduction in the available momentum for vertical transport. Smaller near-surface gust factors are expected at small radii as shown by Schroeder et al. (2009). The two results suggest that current gust envelopes employed in design standards may be a conservative estimate of the expected wind gusts. The results may also help explain the rarity of "extreme" wind gusts (gust factors > 2.0) in hurricane eyewalls.

Technological advances have allowed the hurricane boundary layer to be probed in greater detail. GPS sondes have provided a significant number of vertical profiles for analysis and the use of composite profiles has been useful in evaluating mean structure. Although over 1000 GPS sondes were employed for this study only 55 sondes existed within the dataset that landed within $5 \mathrm{~km}$ of the immediate shoreline. This data void illustrates the continued need for observations at landfall. The VAD derived profiles were useful in filling in the gap and providing information regarding the mean structure and evolution of the wind profile over land. Data are still lacking from high wind regimes at landfall as the peak VAD wind speed within the dataset was only $56 \mathrm{~ms}^{-1}$. Evaluating roughness conditions from the VAD profiles is also quite difficult given the temporal and volumetric averaging associated with the technique. The annulus of radial velocity measurements from which the VAD profile is derived, likely includes a variety of roughness lengths and internal boundary layer influences. Continued work is needed in order to understand the effective roughness regime a VAD wind profile represents. The understanding of how transitional flow regimes influence the vertical wind profile is vital for engineering interests as well as hurricane wind field and risk modeling efforts. The limitations of the current study provide motivation for continued and concentrated efforts to observe tropical cyclones both at sea and at landfall.

\section{Acknowledgements}

Financial support for the various data collection and analysis efforts has been provided by the National Science Foundation Grant DGE-0221688, Department of Energy cooperative agreement 
DE-FG36-06G0B6092, the Wind Science and Engineering Research Center at Texas Tech University, and the Department of Geosciences. Thanks are extended to Russell St. Fleur, Nick Carrasco, and Sonia Otero for assistance with the NOAA-HRD GPS dropwindsonde database and $\mathrm{H}^{*}$ Wind.

\section{References}

Amano, T., Fukushima, H., Ohkuma, T., Kawaguchi, S. and Goto, S. (1999), "The observation of typhoon winds in Okinawa by Doppler sodar", J. Wind Eng. Ind. Aerod, 83(1-3), 11-20.

Angell, J.K., Pack, D.H., Hoecker, W.H. and Delver, N. (1971), "Lagrangian-Eulerian time-scale ratios estimated from constant volume balloon flights past a tall tower", Q. J. Roy. Meteor.Soc., 97(411), 87-92.

ASCE (2010), ASCE 7-10 Minimum Design Loads for Buildings and Other Structures.

Black P.G., D’Asaro, E., Drennan, W.M., French, J.R., Niiler, P.P., Sanford, T.B., Terrill, E.J., Walsh, E.J. and Zhang, J.A. (2007), "Air-sea exchange in hurricanes: Synthesis of observations from the coupled boundary layer Air-sea transfer experiment”, B. Am. Meteorol. Soc., 88, 357-374.

Blackwell K.G. (2000), "The evolution of Hurricane Danny (1997) at landfall: Doppler-observed eyewall replacement, vortex contraction/intensification, and low-level wind maxima", Mon. Weather. Rev., 128(12), $4002-4016$.

Bonner, W.D. (1968), “Climatology of the low-level jet”, Mon. Weather. Rev., 96(12), 833-850.

Browning, K.A. and Wexler, R. (1968), "The determination of kinematic properties of a wind field using Doppler radar", J. Appl. Meteorol Clim., 7, 105-113.

Franklin, J.L., Black, M.L. and Valde, K. (2003), "GPS dropwindsonde profiles in hurricanes and their operational implications", Weather. Forecast., 18, 32-44.

Hock, T.F. and Franklin, J.L. (1999), "The NCAR GPS dropwindsonde”, B. Am. Meteorol. Soc., 80(3), 407-420.

Hsu S.A., Meindl E.A. and Gilhousen D.B. (1994), "Determining the power-law wind- profile exponent under near-neutral stability conditions at sea", J. Appl. Meteorol Clim., 33(6), 757-765.

Jorgensen, D.P. (1984), "Mesoscale and convective scale characteristics of mature hurricanes, Part II: Inner core structure of Hurricane Allen (1980)", J. Atmos. Sci., 41, 1287-1311.

Kepert, J.D. (2001), "The dynamics of boundary layer jets within the tropical cyclone core, Part I: Linear theory", J. Atmos. Sci., 58, 2469-2483.

Kepert, J.D. and Wang, Y. (2001), "The dynamics of boundary layer jets within the tropical cyclone core. Part II: Non-linear enhancement", J. Atmos. Sci., 58, 2469-2483.

Kepert, J.D. (2006a), "Observed boundary layer wind structure and balance in the hurricane core. Part I: Hurricane Georges”, J. Atmos. Sci., 63, 2169-2193.

Kepert, J.D. (2006b), "Observed boundary layer wind structure and balance in the hurricane core. Part II: Hurricane Mitch", J. Atmos. Sci., 63, 2169-2193.

Klazura, G.E. and Imy, D.A. (1993), "A description of the initial set of analysis products available from the NEXRAD WSR-88D system”, B. Am. Meteorol. Soc., 74(7), 1293-1311.

Knupp, K.R. Walters, J. and Biggerstaff, M. (2006), "Doppler radar and profiler observations of boundary layer variability during the landfall of Tropical Storm Gabrielle", J. Atmos. Sci., 63, 243-251.

Lorsolo, S., Schroeder, J.L., Dodge, P. and Marks, F. (2008), "An Observational study of hurricane boundary layer small-scale features", Mon. Weather. Rev., 136, 2871-2893.

Moon, I.J., Ginis, I. and Hara, T. (2004), "Effect of surface waves on air-sea momentum exchange. Part II: Drag coefficient under tropical cyclones", J. Atmos. Sci., 61, 2334-2348.

Pielke, R.A. Jr., Gratz, J., Landsea, C.W., Collins, D., Saunders, M.A. and Musulin, R. (2008), "Normalized hurricane damage in the United States: 1900-2005", Nat. Hazards., 9(1), $29-42$.

Powell, M.D., Houston, S.H., Amat, L.R. and Morisseau-Leroy, N. (1998), "The HRD real-time hurricane wind analysis system", J. Wind Eng. Ind. Aerod., 77-78, 53-64.

Powell, M.D., Vickery, P.J. and Reinhold, T.A. (2003), "Reduced drag coefficients for high wind speeds in tropical cyclones", Nature, 422, 279-283. 
Powell, M.D. (2007), Final report to the NOAA Joint Hurricane Testbed: Drag coefficient distribution and wind speed dependence in tropical cyclones, NOAA Hurricane Research Division.

Powell, M.D., Uhlhorn, E. and Kepert, J.D. (2009), "Estimating maximum surface winds from hurricane reconnaissance measurements", Weather Forecast., 24, 868-883.

Schroeder, J.L., Edwards, B.P. and Giammanco, I.M. (2009), "Observed tropical cyclone wind flow characteristics", Wind Struct., 12(4), 347-379.

Schwendike, J. and Kepert, J.D. (2008), "The boundary layer winds in Hurricanes Danielle (1998) and Isabel (2003)", Mon. Weather. Rev., 136(8), 3168-3192.

Stull, R.S. (1988), An Introduction to Boundary Layer Meteorology, Kluwer Academic.

Vickery, P.J., Wadhera, D., Powell, M.D. and Chen, Y. (2009), "A hurricane boundary layer and wind field model for use in engineering applications", J. Appl. Meteorol. Clim, 48, 381-405.

Willoughby, H.E. and Chelmow, M. (1982), "Objective determination of hurricane tracks from aircraft observations", Mon. Weather. Rev., 110(9), 1298-1305.

Willoughby, H.E. (1990), "Gradient balance in tropical cyclones", J. Atmos. Sci., 47(2), 265-274.

Wright, C.W., Walsh, E.J., Vandemark, D., Krabill, W.B., Garcia, A., Houston, S.H., Powell, M.D., Black, P.G. and Marks, F.D. (2001), "Hurricane directional wave spectrum spatial variation in the open ocean", J. Phys. Oceanogr., 31, 2472-2488.

Wurman, J. and Winslow, J. (1998), "Intense sub-kilometer scale boundary layer rolls observed in Hurricane Fran”, Science, 280(5363), 555-557.

$J H$ 